\title{
INSPEC, UNA BASE DE DATOS ESENCIAL PARA LA FÍSICA Y LA INGENIERÍA
}

\section{Charles Martínez}

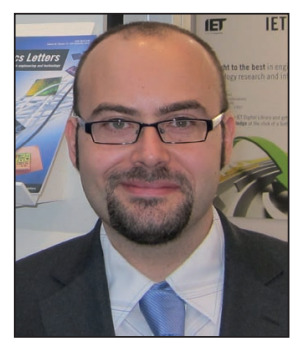

Charles Martínez es doctor en física por la Universidad de Nottingham, y licenciado en física aplicada por la Universidad de Portsmouth, ambas en el Reino Unido. Administra las relaciones con clientes de Inspec de Europa Occidental. Fue indexador de física de estado sólido y ciencias de materiales para la base de datos Inspec durante 5 años. Ha impartido cursos de formación por toda Europa.

The IET

Michael Faraday House, Six Hills Way Stevenage, SG1 2AY, Reino Unido cmartinez@theiet.org

\section{Resumen}

La base de datos Inspec contiene más de 12 millones de registros bibliográficos de física, electrónica, informática e ingeniería. Aunando tradición, calidad y exhaustividad no tiene rival en su temática en el mercado actual de la información. Desde el punto de vista estrictamente documental Inspec se considera como uno de los sistemas mejor diseñados por su equilibrada y eficaz indexación, apoyada en sus valiosos clasificación y thesaurus. Se presenta una breve descripción de su contenido, campos de valor añadido, así como sus nuevos desarrollos.

\section{Palabras clave}

Base de datos, Referencias bibliográficas, Física, Electrónica, Informática, Documentación, Ingeniería.

\section{Title: Inspec, an essential database for physics and engineering}

\section{Abstract}

The Inspec database contains more than 12 million bibliographic records on physics, electronics, computer science and engineering. Combining tradition, quality and thoroughness it is unmatched in its topic area in the current information market. From a strictly documentary point of view, Inspec is considered as one of the best-designed systems for its balanced and effective indexing, based on its well known classification and thesaurus. A brief description of its contents, value-added fields and new developments is presented.

\section{Keywords}

Database, Bibliographic references, Physics, Electronics, Computer science, Documents, Engineering.

Martínez, Charles. Inspec, una base de datos esencial para la física y la ingeniería. El profesional de la información, 2011, septiembre-octubre, v. 20, n. 5, pp. 577-582.

http://dx.doi.org/10.3145/epi.2011.sep.13

\section{Introducción}

Inspec es una base de datos realmente imprescindible para la física, ingenierías eléctrica y electrónica, computación y control, tecnologías de la información, así como para la ingeniería mecánica y de producción. Fue creada en 1969 a partir de los Science Abstracts Journals publicados por la Institution of Electrical Engineers (IEE) ${ }^{1}$ de Londres desde 1898.

Inspec se puede consultar a través de varios distribuidores (tabla 1).

Contiene más de 12 millones de registros bibliográficos, con un crecimiento anual de 670.000 (figura 2), lo que represen- ta un ritmo de 1.800 diarios, festivos incluidos. Indexa 5.000 publicaciones científicas y técnicas, y unas 3.000 actas de congresos y conferencias cada año.

A los temas tradicionales PEC añadió en 2004 la ingeniería de producción y en 2005 la ingeniería mecánica. Adicionalmente ofrece una amplia gama de temas multidisciplinares como biofísica, oceanografía, nanotecnología y ciencias de materiales.

Una de sus secciones más interesantes para los lectores de EPI es la de Tecnología de la información, también con más de 40 años de presencia en la base de datos, que ha recogido todo lo publicado sobre automatización de bibliotecas y 


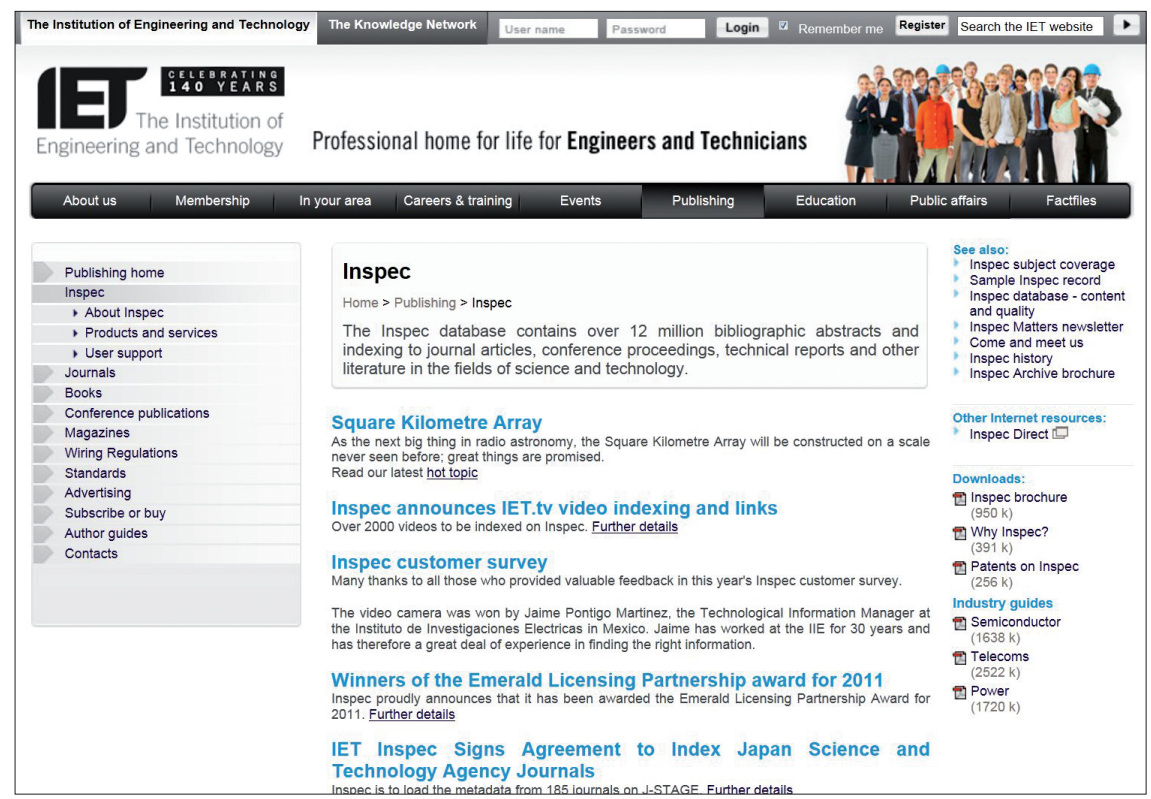

Figura 1. Home de la base de datos Inspec, http://www.theiet.org/publishing/inspec/

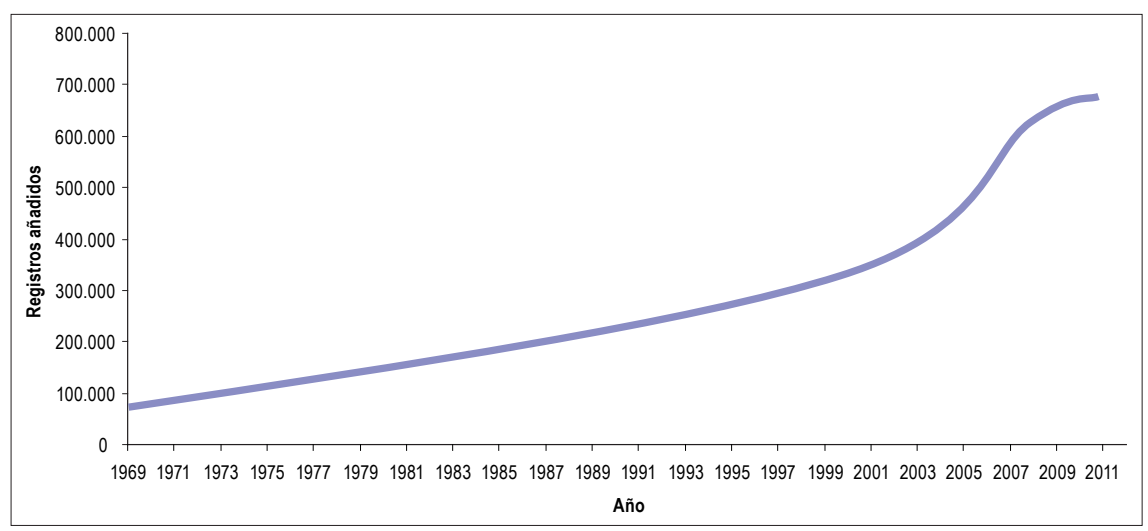

Figura 2. Desde 1969 cada año se supera la cifra de registros añadidos la base de datos. como mínimo 1 descriptor a cada registro, aunque el promedio es de 5 ó 6 , siempre al nivel más específico posible.

\section{Términos no-controlados}

Como se sabe, son palabras o frases que expresan conceptos adicionales -aunque importantes- del documento, el cual no quedaría completamente definido sólo con los descriptores. Muchas veces no aparecen en el título ni en el resumen del documento. Añadidos por los indizadores de Inspec, no siguen ningún estándar o regla, y es frecuente que se aprovechen las mismas palabras clave que figuran en el artículo. En el distribuidor Dialog se llaman Identifiers.

\section{Son útiles para buscar:}

- temas nuevos para los que aún no se ha acordado crear el correspondiente término controlado;

- sustancias químicas orgánicas;

- sustancias químicas inorgánicas (en documentos anteriores a 1987);

- palabras que poseen un doble significado, común y técnico;

- acrónimos y nombres comerciales.

\section{Clasificación Inspec}

Está dividida en cinco secciones (figura 3 y tabla 2 ):

\section{A. Física}

B. Ingeniería eléctrica y electrónica

C. Computación y control

D. Tecnología de la información

E. Ingeniería mecánica y de producción

En las búsquedas la clasificación se puede aplicar a cualquier nivel, desde el más amplio (letra de la sección +1 dígito) al más especifico (seis dígitos).

Comunicaciones en actas

$8 \%$

Comunicaciones publicadas en revistas

Resto

$2 \%$

\section{Tesauro Inspec}

Su última edición (2010) lista 18.400 términos, de los cuales 9.600 son descriptores o términos de indexación controlados, con sus relaciones correspondientes. Inspec asigna

\section{A. Física \\ B. Ingeniería eléctrica y electrónica \\ C. Computación y control \\ D. Tecnología de información \\ E. Ingeniería mecánica y de producción}

Figura 3. Contenido por temas

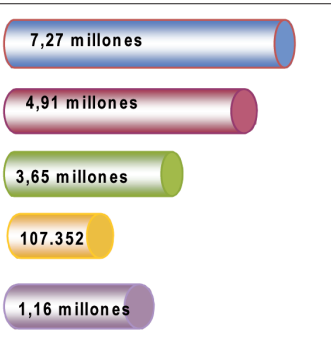

1,16 millones

\begin{tabular}{|l|c|c|c|c|}
\hline \multicolumn{1}{|c|}{ Distribuidor } & $\begin{array}{c}\text { Actuali- } \\
\text { zación }\end{array}$ & Años & $\begin{array}{c}\text { Suscrip- } \\
\text { ción }\end{array}$ & $\begin{array}{c}\text { Pago } \\
\text { según } \\
\text { uso }\end{array}$ \\
\hline DataStar & Semanal & $1898-$ & Sí & Sí \\
\hline Dialog & Semanal & $1898-$ & Sí & Sí \\
\hline Ebsco & Semanal & $1898-$ & Sí & \\
\hline Engineering index & Semanal & $1898-$ & Sí & \\
\hline WTi & Semanal & $1969-$ & Sí & Sí \\
\hline $\begin{array}{l}\text { Thomson Web of } \\
\text { Knowledge }\end{array}$ & Semanal & $1898-$ & Sí & \\
\hline Ovid & Semanal & $1898-$ & Sí & \\
\hline Questel.Orbit & Semanal & $1969-$ & Sí & Sí \\
\hline STN & Semanal & $1898-$ & Sí & Sí \\
\hline Thomson Innovation & Semanal & $1898-$ & Sí & \\
\hline
\end{tabular}

Tabla 1. Proveedores de Inspec 


\begin{tabular}{|l|}
\hline \multicolumn{1}{|c|}{ Clasificación Inspec } \\
\hline A - Física \\
A0 General \\
A1 Física de partículas \\
A2 Física nuclear \\
A3 Molecular y atómica \\
A4 Física fundamental \\
A5 Plasmas y descargas \\
A6 Estado sólido, no-electrónico \\
A7 Estado sólido, electrónico \\
A8 Física multidisciplinar \\
A9 Geofísica y astronomía \\
B - Ingeniería eléctrica y electrónica \\
B0 General, matemáticas y materiales \\
B1 Circuitos \\
B2 Componentes, dispositivos electrónicos \\
B3 Dispositivos magnéticos y materiales \\
B4 Optoelectrónica \\
B5 Campos electromagnéticos \\
B6 Comunicaciones \\
B7 Instrumentos y aplicaciones \\
B8 Potencia \\
C - Computación y control \\
C0 Gestión general \\
C1 Sistema y teoría de control \\
C3 Tecnología de control \\
C4 Teoría numérica y de computación \\
C5 Hardware de computadoras \\
C6 Software de computadoras \\
C7 Aplicaciones \\
D - Tecnología de información \\
D1 Gestión \\
D2 Aplicaciones \\
D3 Sistemas generales \\
D4 Automoción de oficinas / comunicaciones \\
D5 Automoción de oficinas / computación \\
E- Ingeniería mecánica y de producción \\
E0 Temas generales de ingeniería mecánica y de producción \\
E1 Fabricación y producción \\
E2 Ingeniería mecánica \\
E3 Sectores industriales \\
\hline
\end{tabular}

Tabla 2. Clasificación Inspec a nivel 2 dígitos

- los códigos de clasificación más amplios (como B7* o B72*) son particularmente útiles para centrar los resultados obtenidos y aumentar la precisión; pero también al contrario, para guiar a los usuarios ocasionales hacia la parte más apropiada de la base de datos, y que empiecen su búsqueda por ahí.

- los códigos más específicos (como por ejemplo, b7230J para biosensores) son cómodos para cuando se busca repetidamente sobre el mismo tema. Y también para crear alertas (DSI).

\section{Códigos de tratamiento}

Una característica propia de Inspec, no usual en las bases de datos en general, es la asignación de códigos de tratamiento para indicar el planteamiento o enfoque del artículo. Permiten centrar la búsqueda en los registros más relevantes cuando se ha obtenido una gran cantidad de documentos sobre un tema. En total son 9 y los documentos puede tener asignados uno o más de ellos:

Aplicación, Bibliografía, Económico, General o Revisión, Nuevo desarrollo, Práctico, Revisión de producto, Teórico y Experimental.

\section{Sustancias químicas}

Inspec tiene un sistema de indexación controlada para sustancias inorgánicas diseñado para resolver ambigüedades.
Ejemplos:

- Aleaciones o compuestos no-estequiométricos (compuestos con proporciones de elementos variables) que pueden ser representadas de varias formas:

GaAlAs o bien $\mathrm{Ga}_{\mathrm{x}} \mathrm{Al}_{(1-\mathrm{x})}$ As.

- Fórmulas químicas que poseen la misma ortografía que una palabra común en inglés: fosfato de galio $(\mathrm{GaP})$, indio (In), etc.

- Sustancias químicas que poseen las mismas letras y sólo se diferencian por las mayúsculas o minúsculas: Co (cobalto) y CO (monóxido de carbono).

Cada sustancia química posee un indicador de rol:

el = elementos, por ejemplo $\mathrm{Si}$

bin = compuestos binarios (dos componentes), por ejemplo GaAs.

ss = para sistemas (tres o más componentes), por ejemplo $\mathrm{H} 2 \mathrm{SO} 4$

A algunas sustancias se les pueden asignar indicadores de rol especiales:

int = capas emparedadas (interface system)

sur $=$ superficie o sustrato

ads = adsorbato

dop $=$ dopante

Ejemplos:

Si (silicio) es indexado como Si/el

$\mathrm{SiO}_{2}$ (dióxido de silicio) como $\mathrm{SiO}_{2} /$ bin $\mathrm{Si} / \mathrm{bin} \mathrm{O} / \mathrm{bin}$

$\mathrm{H}_{2} \mathrm{SO}_{4}$

$\mathrm{H}_{2} \mathrm{SO}_{4} / \mathrm{ss} \mathrm{SO}_{4} / \mathrm{ss} \mathrm{H}_{2} / \mathrm{ss} \mathrm{O}_{4} / \mathrm{ss} \mathrm{H}_{\text {/ss S/ss O/ss }}$

Si dopado con $\mathrm{P}$

$\mathrm{Si}: \mathrm{P} /$ bin Si/bin P/bin Si/el P/el P/dop

Aleación Cu-Al

$\mathrm{CuAl} /$ bin $\mathrm{Cu} / \mathrm{bin} \mathrm{Al} / \mathrm{bin}$

Si-Au capas emparedadas

Si-Au/int Si/int Au/int Si/el Au/el

GaAlAs

GaAlAs/ss Ga/ss Al/ss As/ss

$\mathrm{Ga}_{\mathrm{x}} \mathrm{A}_{11-\mathrm{x}}$ As

GaAlAs/ss Ga/ss Al/ss As/ss

$\mathrm{Ga}_{0.25} \mathrm{Al}_{0.75} \mathrm{As}$

$\mathrm{Ga}_{0.25} \mathrm{Al}_{0.75} \mathrm{As} / \mathrm{ss} \mathrm{Ga}{ }_{0.25} / \mathrm{ss} \mathrm{Al}_{0.75} / \mathrm{ss} \mathrm{Ga} / \mathrm{ss} \mathrm{Al} / \mathrm{ss}$ As/ss

\section{Indexación de datos numéricos}

Si se desea encontrar trabajos, por ejemplo, sobre centrales eléctricas que generen energía entre 20 y $30 \mathrm{MW}$ de potencia, nos encontramos que en los artículos originales los valores pueden figurar como $29.2 \mathrm{MW} ; 29,2 \mathrm{MW}$; 27500 KW; 25 megawatts; 29 MWatt; etc. En Inspec se ha normalizado la forma de expresar esas cantidades usando la notación de coma flotante (floating point) y el formato cantidad+decimales o ceros y unidad de medición (según el Sistema Internacional). Así, por ejemplo, una potencia de 25 megavatios (25.000.000) se escribe 2.5E+07W; valores entre $30 \mathrm{~Hz}$ y $18 \mathrm{KHz}$ se escriben $3.0 \mathrm{E}+01$ to $1.8+04 \mathrm{~Hz}$; una radiación nuclear de 0,0024 sieverts se escribe 2.4E-03Sv 


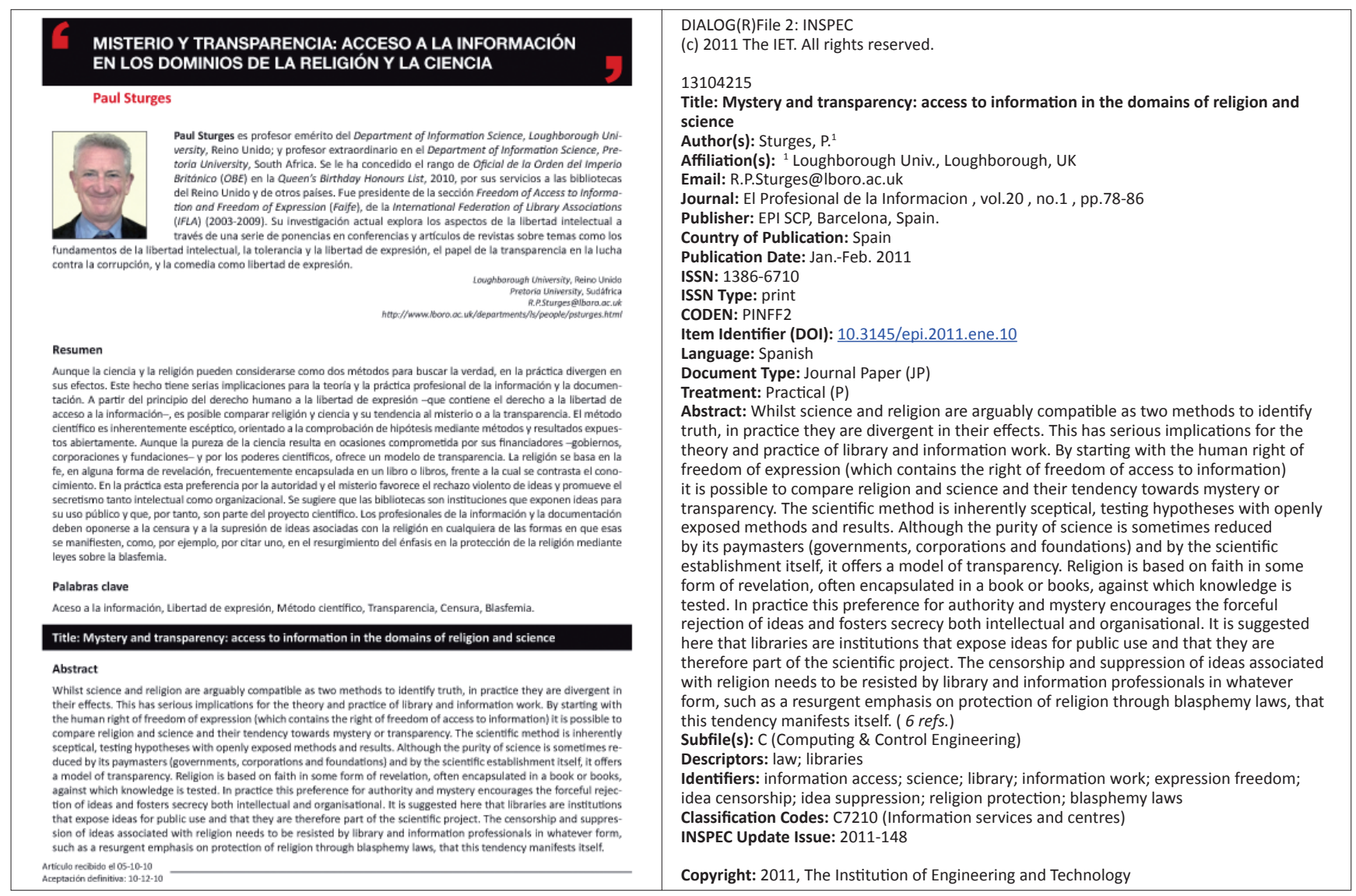

Figura 4. Artículo de El profesional de la información y su correspondiente registro en Inspec (versión Dialog). Haciendo clic sobre el DOI se accede al pdf del documento ubicado en la web http://elprofesionaldelainformacion.metapress.com

La base de datos permite buscar por rangos numéricos expresados de esa forma, en un campo específico de las referencias.

\section{Indexación de objetos astronómicos}

Desde 1995 figuran en un campo de búsqueda aparte, y se pueden buscar tanto por nombre como por número.

\section{- Acrónimos de nombres}

Se usan las abreviaciones de tres letras aprobadas por la International Astronomical Union (IAU). Por ejemplo, la constelación Gran Nube de Magallanes se abrevia LMC (acrónimo de Large Magellanic Cloud). Los objetos dentro de constelaciones se indican con R Sct, por ejemplo R Sct HDW.

- Acrónimos del catálogo NGC

Se usan acrónimos y números del New general catalogue of nebulae and clusters of stars (NGC). El número puede ser secuencial, como NGC 204 o puede representar la posición aproximada en el cielo, normalmente en términos de ascensión recta y declinación (como PSR 1913+16) o de coordenadas galácticas (por ejemplo G345.01+1.79).

- Información de posición

Por ejemplo $013022+30233$

\section{Códigos IPC (International patent classification)}

Inspec ha emparejado su clasificación con la IPC de la WIPO (World Intellectual Property Organization). Los códigos IPC, que figuran en la base de datos desde 1969, son muy útiles para los investigadores de patentes, que de esta forma pueden buscarlas usando la estructura de códigos que les resulte más familiar. Los esquemas IPC e Inspec no cubren los mismos conceptos exactamente, y por ello el grado de precisión de las búsquedas usando IPC depende de la relaciones entre ambos esquemas y de cada tema específico, pero el eventual pequeño ruido documental marginal que se produce puede ayudar a hacer nuevos descubrimientos. Los códigos IPC figuran en un $75 \%$ de los registros.

Desde este sitio puede bajarse una lista de los códigos IPC utilizados por Inspec:

http://www.theiet.org/publishing/inspec/about/records/ IPC.cfm

\section{Guías de usuario}

Disponibles en castellano para los diferentes distribuidores de la base de datos:

http://www.theiet.org/publishing/inspec/support/docs/ index.cfm

\section{Inspec archive}

Es una base de datos retrospectiva que da acceso a 70 años de investigación científica y tecnológica (desde 1898 a 1968). Se trata de la colección digitalizada completa de los Science abstracts journals: 800.000 registros de física, ingeniería eléctrica, electrónica e ingeniería de control.

Aparte de encontrar referencias a los materiales originales de renombrados autores como Marconi, Marie Curie o Einstein, los usuarios del archivo pueden encontrarse con una 
bella durmiente, es decir una idea estudiada hace años que en su momento fue descartada pero que con la evolución tecnológica podría ser de interés en el día de hoy. La solución a los problemas es a menudo atemporal, y muchas de las investigaciones registradas en el Archive todavía son relevantes para los investigadores modernos. A continuación se incluyen algunos ejemplos de tecnologías descritas en el Archive que hoy se vuelven a usar para intentar re-descubrir soluciones a temas candentes:

\section{Innovación frugal}

Este tipo de innovación se está volviendo cada vez más importante en el clima económico actual. Las eco-innovaciones se diseñan para ser baratas, robustas y fáciles de usar. Se han denominado también "innovaciones de marcha atrás" (reverse) o "basadas en restricciones". Se caracterizan por limitar el uso de materias primas y por tratar de minimizar su impacto sobre el medio ambiente.

Este tipo de innovación requiere replantearse todo desde cero, para así hallar soluciones simples y efectivas, y en estos casos puede ser eficaz investigar los principios.

\section{Cosechar energía}

Energy harvesting es el aprovechamiento de las energías naturales (solar, térmica, eólica, cinética, gradientes de salinidad, etc.) que son gratuitas y no contaminan.

La búsqueda de soluciones para aminorar el carbon footprint, o restos de gases de efecto invernadero $\left(\mathrm{CO}_{2}\right)$ producidos por la actividad humana, también ha conducido a tratar de aprovechar energías desperdiciadas. Se trata de capturar restos de basura energética desprendida de motores en funcionamiento; de las instalaciones de calefacción y agua caliente de los edificios; del campo electromagnético urbano creado por la telefonía móvil y las emisoras de radiotelevisión; del movimiento del ser humano; de las vibraciones de las máquinas...

La energía capturada se almacena en células que permiten operar pequeños dispositivos electrónicos.

\section{Células de combustible}

Están ganando mucha popularidad como fuente de energía sin emisiones, pues convierten el combustible en energía eléctrica desprendiendo únicamente calor y agua. Existen ya muchos tipos de ellas. En el Inspec Archive se encuentran los registros que describen las investigaciones originales, particularmente en el periodo desde los finales de los años 40 hasta los 60 . Por los 60 fue cuando más se renovó el interés en esta tecnología para su uso en las misiones espaciales Gemini y Apollo.

\section{Transmisión inalámbrica de potencia}

La transmisión de electricidad sin la necesidad de cables es hoy en día más que nunca un hot topic, pues podría ser usada en dispositivos portátiles en el hogar y en la oficina.

\section{Frenos regenerativos}

Son dispositivos que permiten reducir la velocidad de un vehículo transformando parte de su energía cinética en energía eléctrica. Se aplicaron a las locomotoras eléctricas ya en los años 30 y actualmente se pueden encontrar en vehículos eléctricos y en motores de combustión interna convencionales. El Archivo Inspec contiene registros que describen las primeras aplicaciones de la tecnología de freno regenerativo en vehículos y maquinaria.

\section{Inspec en 2011}

\section{Citas}

Para mejorar la recuperación de registros, Inspec ha empezado a añadir citas de artículos publicados desde finales del 2010. La funcionalidad dependerá de la plataforma en que se utilice la base de datos, pero cada cita tendrá un enlace a su registro Inspec al igual que al texto completo correspondiente.

\section{Vídeos}

La base de datos Inspec indexa los más de 3.300 vídeos de carácter científico producidos por The IET, que pueden consultarse en la web:

http://tv.theiet.org/index.cfm

Tesis doctorales de la Universidad Complutense de Madrid

Inspec ha empezado a indexar las primeras 50 tesis doctorales de las Facultades de Física y de Informática de la Universidad Complutense de Madrid.

Las instituciones interesadas en que Inspec indice sus tesis pueden solicitarlo a:

inspec@theiet.org

\section{Bases de datos competidoras}

En el mercado existen varias bases de datos que también tratan los temas cubiertos por Inspec. No entra dentro de los objetivos de este artículo compararlas (para ello véanse artículos como el de Salisbury; Gupta, 2004) pero para que el lector tenga una idea de la oferta sí creemos imprescindible al menos listarlas. Incluimos la propia base de datos Inspec:

Inspec (inicialmente Information service for physics, electronics, and computing)

12 millones de referencias, 1969-

The Institution of Electrical Engineers

http://www.theiet.org/publishing/inspec

\section{Inspec archive}

800.000 referencias, $1898-1968$

The Institution of Electrical Engineers

http://www.theiet.org/publishing/inspec/products/range/ archive.cfm

\section{Spin (Searchable physics information notices)}

2,35 millones de referencias, American Institute of Physics http://scitation.aip.org

\section{Solid state and superconductivity abstracts}

1,2 millones de referencias, 1981- 
Cambridge Scientific Abstracts

http://www.csa.com/factsheets/solid-state-set-c.php

\section{Computer and information systems abstracts}

1,5 millones de referencias, 1981 -

Cambridge Scientific Abstracts

http://www.csa.com/factsheets/computer-set-c.php

\section{Aerospace \& high technology database}

Contiene los antiguos International aerospace abstracts (IAA) y Scientific and technical aerospace reports (STAR) de la NASA (1962-1993) más las actualizaciones de CSA.

8,4 millones de referencias, 1962 -

Cambridge Scientific Abstracts

http://www.csa.com/factsheets/aerospace-set-c.php

\section{Inis (International nuclear information system)}

3,3 millones de referencias, 1970

International Atomic Energy Agency

http://www.iaea.org/inis

\section{Engineering index (Compendex)}

12 millones de referencias (35\% de ingeniería eléctrica), 1970-

Elsevier

http://www.ei.org/compendex

\section{Engineering index backfile}

1,7 millones de referencias, 1884-1969

Elsevier

http://www.ei.org/compendex

\section{Chemical abstracts}

34 millones de referencias, 1840-

American Chemical Society

http://www.cas.org/expertise/cascontent/index.html

La lista debería completarse con las secciones correspondientes de bases de datos multidisciplinares como $\mathrm{SCl}$ (Thomson Reuters), Scopus (Elsevier), Pascal (Inist) e ICYT (CSIC), así como bases de datos de patentes (Derwent-World patent index, Inpadoc...), bdds de tesis, de actas de conferencias...

\section{Nota}

1. En 2006 el IEE cambió el nombre por IET (Institution of Engineering and Technology).

\section{Bibliografía}

Dess, Howard M. "Inspec via Engineering Village (EV)". Issues in science \& technology librarianship, 2007, Winter, n. 50, p. 4.

Harris, Siân. "IET launches online Inspec platform". Research information, 2008, Feb/Mar, n. 34, pp. 28-28.

"Inspec science abstracts: 100 years, 1898-1998". International information communication and education, 1997, Sept., v. 16, n. 2, pp. 209-214

Kumar, Anil; Kademani, B. S.; Kumar, Vijai. "Web-resources in Inspec database: a scientometric mapping". Srels journal of information management, 2008, June, v. 45, n. 2, pp. 197208.

Prakasan, E. R.; Sagar, Anil; Kumar, Anil; Kalyane, V. L.; Kumar, Vijai. Inspec database analysis for Knowledge Management records.

http://eprints.rclis.org/handle/10760/7263

Salisbury, Lutishoor. "Inspec on InspecDirect and on Engineering Village". Charleston advisor, 2008, April, v. 9, n. 4, pp. 5-11.

Salisbury, Lutishoor; Gupta, Usha. "A comparative review of Inspec on EbscoHost, Engineering Village (EV2), and Institute for Scientific Information (ISI)". Charleston advisor, 2004, Jul, v. 6, n. 1, pp. 5-11.

"The IET announces launch of Inspec Direct". Managing information, 2007, Dec., v. 14, n. 9, pp. 25-25.

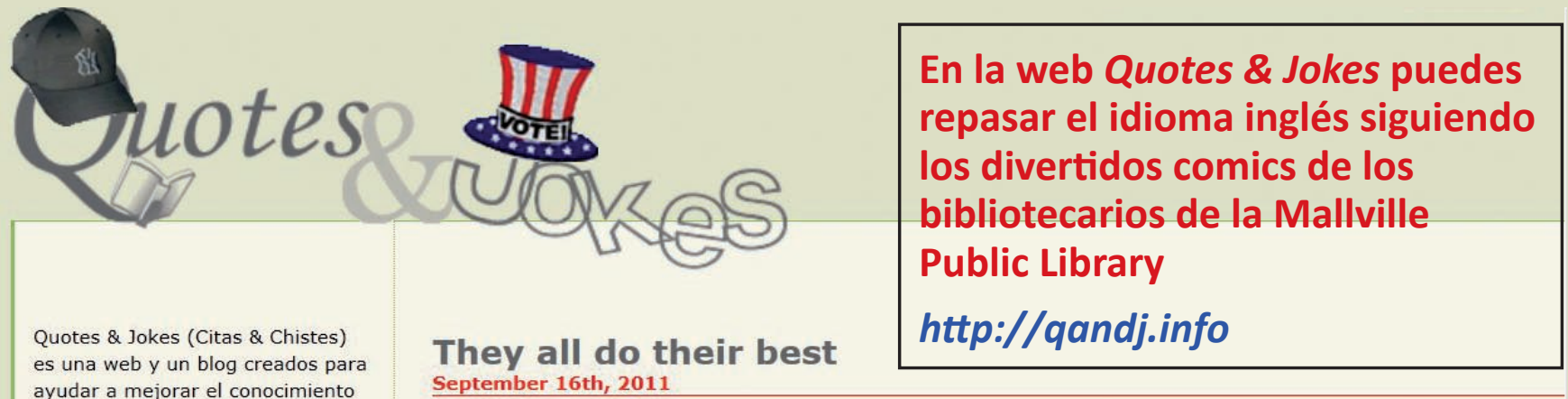
de idioma inglés entre las personas castellanoparlantes $y$, entre ellas, especialmente a los bibliotecarios, documentalistas y expertos en información.

Leer complete

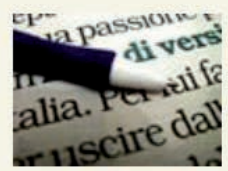

Dear colleagues,

Mallville librarians have to help techno-illiterates, and not only those who are patrons.
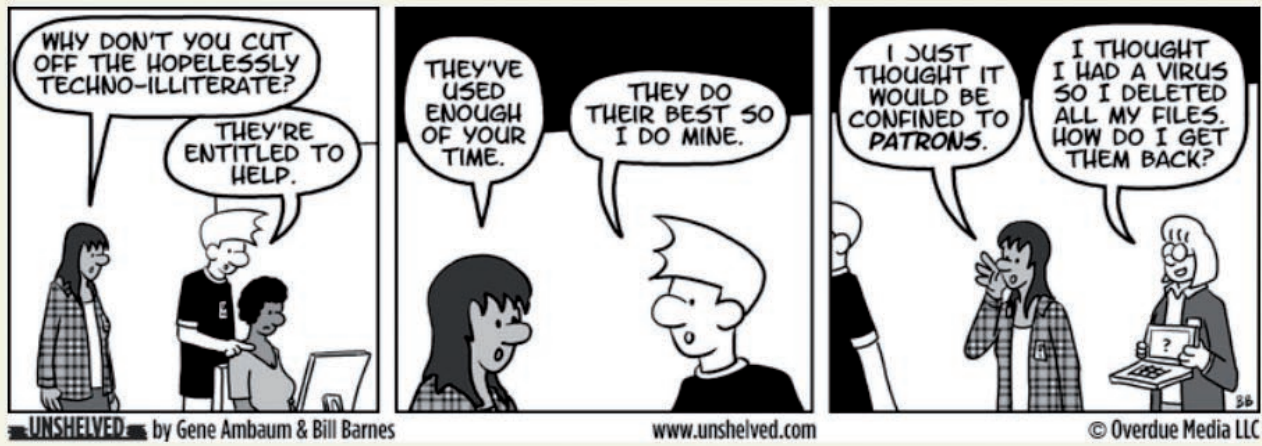

http://www.unshelved.com/2011-9-6 